\title{
The Nanos gradient in Drosophila embryos is generated by translational regulation
}

\author{
Anupama Dahanukar and Robin P. Wharton ${ }^{1}$ \\ Department of Genetics, Howard Hughes Medicál Institute, Duke University Medical Center, \\ Durham, North Carolina 27710 USA
}

\begin{abstract}
Abdominal segmentation in the Drosophila embryo is governed by a gradient of Nanos (Nos) emanating from the posterior pole. This gradient is derived from translation of the nos mRNA that is localized in the pole plasm; in contrast, unlocalized nos mRNA is translationally repressed. Here we define the essential signals in the 3' untranslated region (UTR) of nos mRNA. Deletion of a 184-nucleotide translational control element (TCE) from the 3' UTR leads to the derepression of nos mRNA in the bulk cytoplasm and the development of lethal anterior defects. Furthermore, a minimal mRNA containing essentially only the TCE in its 3' UTR rescues nos ${ }^{-}$embryos to adulthood. The TCE is also sufficient to confer on maternal torso mRNA all three aspects of nos mRNA regulation: translational repression in the bulk cytoplasm, localization to the pole plasm, and translational activation at the posterior pole. These three phenomena are coupled intimately, as mutations in a pair of CUGGC pentamers within the TCE simultaneously abrogate all three regulatory events. This coupling suggests a model in which the polarized distribution of nos protein is generated primarily by translational control and that nos mRNA localization is a byproduct of this regulation, at least in part.
\end{abstract}

[Key Words: Translation; localization; nanos mRNA; Drosophila; development]

Received July 18, 1996; revised version accepted August 23, 1996.

The initial steps in specification of the antero-posterior axis of the Drosophila embryo are regulated by graded signals emanating from each pole of the egg (NüssleinVolhard et al. 1987; Driever 1993; St. Johnston 1993). These graded signals, Bicoid (Bcd) protein at the anterior and Nos protein at the posterior, are generated by translation of localized mRNA precursors. Thus, genesis of these morphogenetic gradients depends critically on the mechanisms that restrict the subcellular localization and translation of mRNA.

$b c d$ mRNA is tightly localized to the anterior pole of the prospective embryo during oogenesis such that none is detectable elsewhere in the egg (Macdonald and Struhl 1988; St. Johnston et al. 1989). As is the case for many maternal messages, translation of bcd mRNA is repressed during oogenesis. Following fertilization, the poly(A) tail of $b c d$ mRNA is extended (Sallés et al. 1994), translation commences, and Bcd protein accumulates in a gradient in the anterior of the preblastoderm embryo (Driever and Nüsslein-Volhard 1988a). Although the signals that mediate the localization and translation regulation of $b c d$ mRNA have not been defined precisely (but see Macdonald et al. 1993; Ferrandon et al. 1994; Sallés et al. 1994), it is clear that these two regulatory events

${ }^{1}$ Corresponding author. can be uncoupled. Genetic analyses have shown that delocalized $b c d$ mRNA is translated normally (Driever and Nüsslein-Volhard 1988b) and that $b c d$ mRNA that is not derepressed following egg activation remains localized (Lieberfarb et al. 1996).

In contrast, localization and translational regulation are coupled for two mRNA determinants that govern posterior development. Normal abdominal segmentation is governed (in part) by the successive action of Oskar (Osk) and Nos. During the early stages of oogenesis, osk mRNA is distributed throughout the cytoplasm of the maturing oocyte; shortly thereafter, a subset of the osk mRNA is localized to the posterior pole of the maturing oocyte (Ephrussi et al. 1991; Kim-Ha et al. 1991, 1993). Recent experiments have shown that the unlocalized osk mRNA is subject to translational repression that is mediated by sites within its $3^{\prime}$ untranslated region (UTR) (Kim-Ha et al. 1995). This repression is relieved at the posterior pole with the result that osk protein is found only in the prospective pole plasm $(\mathrm{Kim}-\mathrm{Ha}$ et al. 1995; Markussen et al. 1995; Rongo et al. 1995).

nos mRNA is localized to the posterior pole of the maturing oocyte during the final stages of oogenesis, after the polarity of the egg chamber has broken down (Theurkauf 1994; Wang et al. 1994). This localization is inefficient; at fertilization, much of the nos mRNA is 
distributed throughout the bulk cytoplasm of the egg and the remainder is localized to the specialized pole plasm at the posterior pole at a somewhat higher concentration. Only the nos mRNA at the posterior pole is translated (Gavis and Lehmann 1994), giving rise to a transient gradient of Nos protein emanating from the pole. The Nos gradient is thought to initiate abdominal patterning by regulating the translation of hunchback $(h b)$ mRNA in collaboration with other factors (Wharton and Struhl 1991; Murata and Wharton 1995).

The localization and translational regulation of nos mRNA depends both on components of the pole plasm such as osk and on signals in its $3^{\prime}$ UTR. Study of various hybrid mRNAs has revealed that the nos 3' UTR contains signals that can direct localization to the posterior pole or translational repression in the bulk cytoplasm (Gavis and Lehmann 1992, 1994; Gavis et al. 1996). These observations have led to the view that the anchoring of a subset of nos mRNA to components of the pole plasm plays a key role in the generation of embryonic polarity. Specifically, the pole plasm has been described as a privileged compartment in which a repressor that otherwise turns off translation of nos mRNA /e.g., in the bulk cytoplasm) is inactive (Gavis and Lehmann 1994). According to this view, the polarized distribution of Nos is generated by the localization-dependent translation of nos mRNA in the pole plasm. However, critical aspects of this model have not been tested. In particular, the relative contributions of localization and translational regulation in restricting Nos activity to the posterior have not been assessed. In addition, it has not been clear whether the translational repression of unlocalized nos mRNA is essential, as is the case for osk mRNA / Kim-Ha et al. 1995).

To address these and other issues, we have defined the regulatory signals in the $3^{\prime}$ UTR of nos mRNA that are required for embryonic development. These studies identify a translational control element (TCE) that is necessary and sufficient (in conjunction with a polyadenylation signal) for nos function in the embryo. Mutational analysis reveals that the critical sites within the TCE consist of redundant, tandem CUGGC pentanucleotides. These pentamer-containing signals are sufficient to mediate the three major aspects of nos mRNA regulation: repression in the bulk cytoplasm, activation in the pole plasm, and localization to the pole plasm. Taken together, these results support a model in which the Nos gradient is generated primarily by differential translational control and not by mRNA localization.

\section{Results}

The 184-nucleotide TCE is necessary and sufficient for nos function

To identify the essential regulatory signals in the nos $3^{\prime}$ UTR, transgenic flies expressing each of the deletion derivatives shown in Figure 1 were prepared. One of the transgenes, $\triangle \mathrm{TCE}$, causes dominant female sterility. All embryos derived from otherwise wild-type females bear-

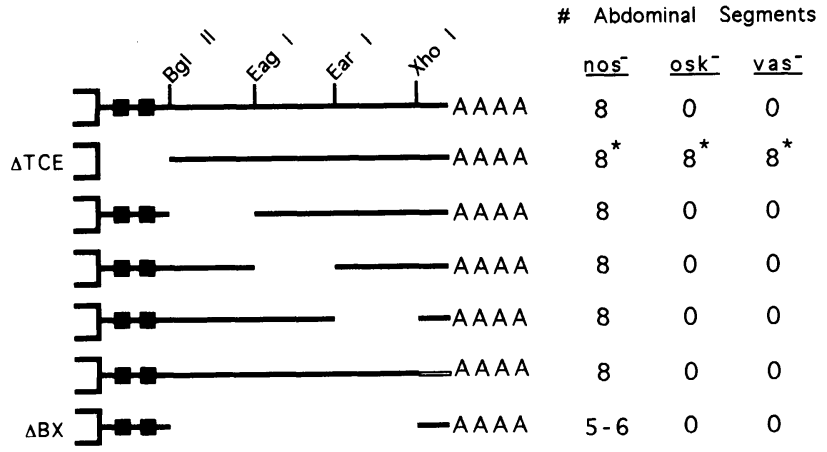

Figure 1. Essential signals within the nos $3^{\prime}$ UTR are confined to the 184-nucleotide TCE. Shown schematically (not to scale) are the structures of mRNAs encoded by various nos derivatives as well as the results of complementation tests at the right. The restriction enzyme sites at the top indicate deletion endpoints, the squares represent the CUGGC-containing pentamers referred to in the text, and the two mRNAs referred to repeatedly in the text $(\triangle T C E$ and $\triangle B X)$ are named at the left. The open bar on the sixth line indicates that nos sequences downstream of XhoI were replaced with sequences encoding part of the 3' UTR and polyadenylation signals of $\alpha$ tub84B (Theurkauf et al. 1986). Each transgene was tested to determine whether it complements the segmentation defects and embryonic lethality of homozygous nos ${ }^{\mathrm{BN}}$, osk ${ }^{166}$, and vas $^{\mathrm{PD}}$ embryos, as indicated. The asterisk indicates that derepression of unlocalized mRNA also causes embryonic lethality and head defects, as described in Fig. 2.

ing the $\triangle \mathrm{TCE}$ transgene die with minor head defects. These defects apparently result from the deregulation of nos mRNA such that ectopic nos activity accumulates in the anterior; evidence supporting this interpretation is discussed below in detail (Fig. 2). An additional indication that the nos( $\triangle \mathrm{TCE})$ mRNA is deregulated comes from analysis of its activity in mutant embryos. Translation of wild-type nos mRNA in the embryo is absolutely dependent on components of the pole plasm, including osk and vasa (vas) (Gavis and Lehmann 1994). However, the nos( $\triangle \mathrm{TCE})$ mRNA evidently is translated in the absence of either osk or vas function; homozygous osk $^{-}$or vas $^{-}$embryos, which develop no abdominal segments if they contain only wild-type nos mRNA, develop a normal complement of 8 abdominal segments if they contain nos( $\triangle \mathrm{TCE})$ mRNA (Fig. 1). Thus, the TCE is necessary for the normal regulation of nos mRNA.

The results of Figure 1 indicate that, with the exception of the TCE, the remainder of the sequences in the $3^{\prime}$ UTR are dispensable for nos function in the embryo. As described above, the ability of various deletion-bearing transgenes to complement the abdominal segmentation defects associated with a nos ${ }^{-}$mutation was assayed. In the absence of nos function, no abdominal segments develop. As shown in Figure 1, a single copy of each transgene (except $\Delta \mathrm{BX}$ ) restores a full complement of $8 \mathrm{ab}$ dominal segments. A single copy of the $\Delta \mathrm{BX}$ transgene partially rescues abdominal development and two copies do so fully. As is the case for nos ${ }^{+}$mRNA, translation of $\operatorname{nos}(\triangle \mathrm{BX})$ mRNA is completely dependent on both osk 


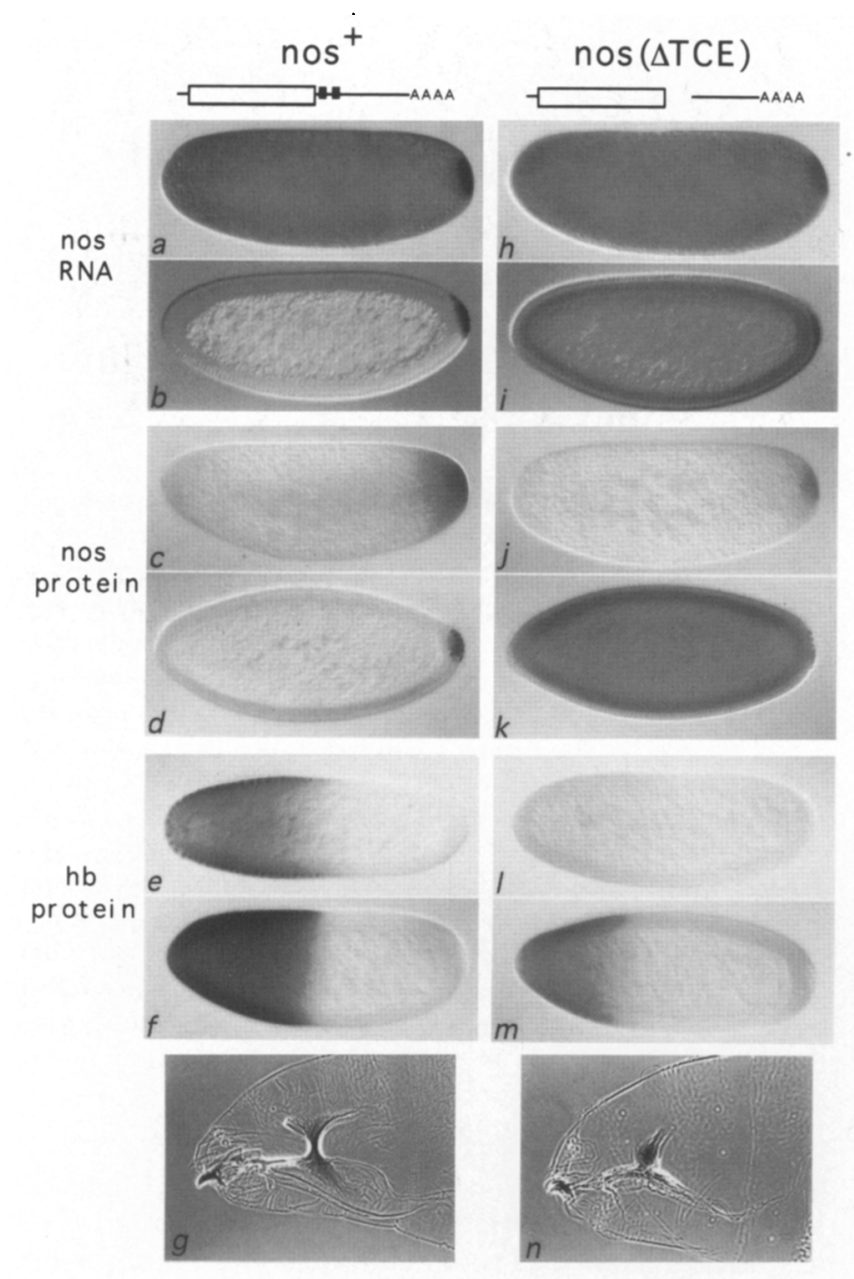

Figure 2. The TCE is required for translational repression of unlocalized nos mRNA and for embryonic viability. Shown are the distributions of nos mRNA (top), nos protein (middle) and $h b$ protein (bottom) in embryos bearing either nos ${ }^{+}$mRNA (left) or nos(ATCE) (right) mRNA. For nos RNA and protein, early and late cleavage stage embryos are shown, above and below, respectively. For $h b$ protein, the younger embryos (above) are in nuclear cycle 10 whereas the older embryos (below) are in late cleavage stage. The $\operatorname{los}^{+}$embryos on the left are $w^{118}$ and thus contain two copies of the wild-type nos gene. The nos $(\Delta \mathrm{TCE})$ embryos are derived nos ${ }^{\mathrm{BN}}$ females bearing a single copy of the appropriate transgene; as a result, all of the nos mRNA and protein in the embryo is derived from the transgene (Wang et al. 1994). In these and all subsequent figures, the anterior of each embryo is to the left and its dorsal surface is at the top. At the bottom are shown phase-contrast micrographs of the heads of $\operatorname{nos}^{+}(\mathrm{g})$ and nos( $\left.\Delta \mathrm{TCE}\right)(\mathrm{n})$ embryos. In the latter, note that structures derived from the most anterior regions of the wild-type fate map (e.g., labral tooth, dorsal bridge) are missing. Also note that the head defects are indistinguishable in nos $(\Delta \mathrm{TCE})$ embryos that are otherwise wild-type, nos $^{-}$, osk $^{-}$, or vas ${ }^{-}$.

and vas (Fig. 1). The 3' UTR of the $\triangle \mathrm{BX}$ mRNA consists only of the TCE, $32 \mathrm{nt}$ flanking the polyadenylation signal, and the poly(A) tail itself. Furthermore, the polyadenylation signal of nos apparently contains no special- ized signals, as it can be substituted by the corresponding sequence from an unregulated mRNA (Fig. 1, line 6; see also Gavis and Lehmann 1994). Thus, the TCE contains all the signals within the $3^{\prime}$ UTR required for normal regulation of nos mRNA; and sequences outside the TCE are not required for its function in the embryo.

\section{The TCE mediates translational repression of unlocalized nos $m R N A$}

The observation that translation of nos( $\triangle \mathrm{TCE})$ mRNA is independent of the usual upstream requirements (e.g., osk function) suggested that it is deregulated. To investigate this further, standard genetic manipulations were performed to prepare homozygous nos ${ }^{\mathrm{BN}}$ mutant females that also contain the nos $(\Delta \mathrm{TCE})$ transgene; in embryos from such females, all the nos mRNA and protein is derived from the transgene (Wang et al. 1994).

We first examined the consequences of removing the TCE on the mRNA. At the beginning of embryogenesis, some of the nos( $\triangle \mathrm{TCE})$ mRNA is localized to the pole plasm whereas the remainder is distributed throughout the bulk cytoplasm, much as is the case for nos ${ }^{+}$mRNA (Fig. 2h). However, in comparison to $\operatorname{nos}^{+}$mRNA, a somewhat lower proportion of nos $(\Delta T C E)$ mRNA appears to be present in the pole plasm, consistent with the idea that the TCE plays a role in normal localization. This is supported by recent results (Gavis et al. 1996) showing that redundant signals for localization exist throughout the nos 3' UTR. As development proceeds in wild-type embryos, the translationally inert unlocalized nos $^{+}$mRNA is degraded while the translationally active $\operatorname{nos}^{+}$mRNA localized to the pole plasm persists (Fig. $2 \mathrm{~b})$. In contrast, unlocalized nos( $\triangle \mathrm{TCE})$ mRNA persists throughout the embryo with the result that nos $(\Delta \mathrm{TCE})$ mRNA is detectable throughout the embryo at stages when nos $^{+}$mRNA is present only in the pole plasm (Fig. $2 \mathrm{~b}, \mathrm{i})$. Based in part on the results described below, we believe the TCE-dependent destabilization of nos mRNA to be a consequence of translational repression, as is the case for other maternal mRNAs (e.g., caudal and $h b$ ) (Macdonald and Struhl 1986; Tautz 1988).

Next, we examined the consequences of removing the TCE on translation of the mRNA. As shown in Figure $2 \mathrm{k}$, the unlocalized nos $(\Delta \mathrm{TCE})$ is translated, and a high level of ectopic Nos accumulates uniformly along the antero-posterior axis. At a similar developmental stage, no Nos is detectable in wild-type embryos except within the pole cells at the posterior (Fig. 2d). Earlier in embryonic development, no ectopic Nos is detectable in $n o s(\Delta \mathrm{TCE})$ embryos; however, the localized nos $(\Delta \mathrm{TCE})$ mRNA is translated, albeit poorly, giving rise to a weak gradient (Fig. 2c,j). This inefficient translation of localized nos( $\triangle \mathrm{TCE})$ mRNA suggests that the TCE may also mediate translational activation in the pole plasm; however, we cannot rule out the possibility that TCE-mediated repression is simply relieved in the pole plasm (Fig. 7 , see below). The main conclusion derived from these observations is that the TCE is required to prevent the translation of unlocalized nos mRNA. 
The ectopic Nos in nos( $\Delta$ TCE) embryos interferes with anterior development by inhibiting the translation of $h b$ and $b c d$ mRNAs. Previous work has shown that, in wildtype embryos, Nos inhibits the translation of maternal $h b$ mRNA in the posterior (Tautz 1988). If expressed at high levels in the anterior of the embryo, Nos blocks the translation of both anterior $h b$ and $b c d$ mRNAs (Wharton and Struhl 1989; Wharton and Struhl 1991). Accordingly, we examined the distributions of $\mathrm{Hb}$ (Fig. 2) and Bcd (data not shown) in nos( $\triangle T C E$ ) embryos. Evidently, the level of ectopic Nos in nos( $\triangle T C E)$ embryos is sufficient only to partially inhibit translation of $b c d$ and $h b$ mRNAs. Thus, although $\mathrm{Hb}$ is virtually absent in cycle 9-10 nos( $\triangle \mathrm{TCE}$ ) embryos (Fig. 2l), the continuous accumulation of $h b$ mRNA and protein in subsequent nuclear cycles leads to the formation of an attenuated $\mathrm{Hb}$ gradient (Fig. $2 \mathrm{~m}$ ). We believe that the reduced levels of $\mathrm{Hb}$ (and Bcd) in such embryos ultimately cause anterior defects and death. In support of this idea, nos( $\triangle \mathrm{TCE}) \mathrm{em}$ bryos that also bear a Nos-resistant $b c d$ mRNA (Wharton and Struhl 1991) survive to adulthood (data not shown).

In summary, we find that: (1) repression of nos $(\Delta \mathrm{TCE})$ mRNA in the bulk cytoplasm is essential for viability, and (2) the TCE contains signals that are required for the repression of unlocalized mRNA. The data in Figure 2 are also consistent with the idea that the TCE plays a role in the activation of localized mRNA; the experiments described below and in Figure 4 (see below) show this to be the case.

The TCE also mediates the destabilization of nos mRNA; however, the following result indicates that this destabilization is a consequence of translational repression. We prepared transgenic flies bearing two different nos-bcd hybrid genes (Gavis and Lehmann 1992) in which the protein coding region of nos is fused to the RNA-localization signals in the 3' UTR of bcd (Macdonald and Struhl 1988) In addition, one of the hybrid mRNAs (nos-TCE-bcd) bears the TCE whereas the other (nos-bcd) does not. As shown in Figure 3, the nosbcd mRNA is localized to the anterior and efficiently translated, giving rise to a high level of Nos which leads to the development of an extreme bicaudal body plan. In contrast, the nos-TCE-bcd hybrid is not detectably translated, and embryos bearing this hybrid develop normal body plans. Significantly, the TCE-bearing hybrid is stable throughout the first several hours of embryogenesis, even though it is not detectably translated. Thus, untranslated nos-TCE- $b c d$ mRNA is relatively stable whereas untranslated nos ${ }^{+}$mRNA is not. We conclude that the TCE mediates primarily translational control, and that signals elsewhere in the untranslated mRNA determine its stability.

\section{The TCE mediates osk-dependent activation and localization}

As described above and in Figure 1, the minimal $\triangle \mathrm{BX}$ nos mRNA derivative, which contains essentially only the TCE in its $3^{\prime}$ UTR, rescues nos ${ }^{-}$embryos to fertile

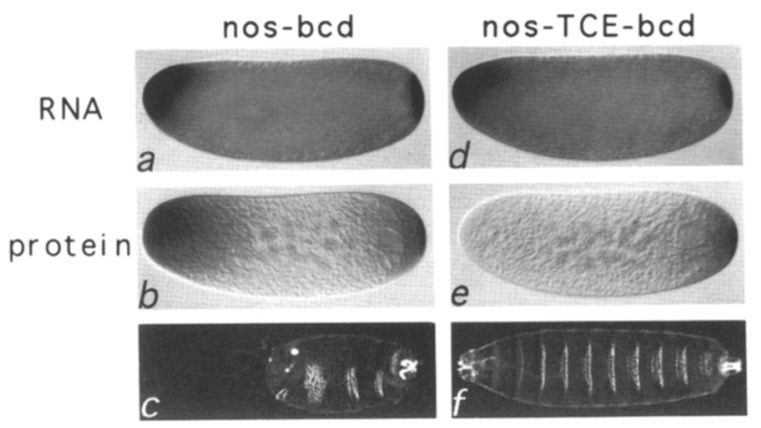

Figure 3. TCE-dependent repression of nos-bcd mRNA. (top) Embryonic distributions of the nos-bcd (left) and nos-TCE-bcd (right) chimeric mRNAs. Note that these chimeric nos mRNAs are localized strictly to the anterior pole /Gavis and Lehmann 1992), and that the endogenous nos ${ }^{+}$mRNA, which is present throughout the bulk cytoplasm and concentrated at higher levels at the posterior pole, is also visible in these preparations. Translation of this endogenous nos ${ }^{+}$mRNA at the posterior pole gives rise to the posterior protein gradients evident in $(b, e)$, whereas translation of the nos-bcd chimera gives rise to the anterior gradient in $(b)$. The segmentation defects in nos-bcd embryos $(c)$ are somewhat greater than those caused by a related transgene (Gavis and Lehmann 1992). We assume this more severely posteriorized phenotype results from the production of higher levels of Nos, much as described by Kim-Ha et al. (1995). Note that a minority of the nos-TCE- $b c d$ embryos develop head defects and die, suggesting that translation of this message is not completely repressed.

adulthood. This raised the possibility that the TCE mediates not only the repression of unlocalized mRNA but that it is also sufficient to mediate the localization and translational activation of nos mRNA in the pole plasm. To test this idea, we constructed a chimeric torso (tor) gene that bears sequences encoding the TCE inserted into those encoding the tor 3' UTR. Germ-line transformants of the resulting tor(TCE) chimeras were generated, and their properties were assayed in the absence of endogenous Tor function as described below.

Tor activity is required at each pole of the embryo for the normal development of the unsegmented terminalia (Casanova and Struhl 1989; Sprenger et al. 1989; Sprenger and Nüsslein-Volhard 1992). Thus, we anticipated that TCE-mediated translational repression might reduce the level of Tor protein below the threshold required for normal development and that, in consequence, tor(TCE) embryos would die. Although, a control tor $^{+}$transgene rescues the lethality of embryos derived from tor ${ }^{-}$females, the tor(TCE) transgene does not. Moreover, examination of the relative levels of RNA and protein in these embryos supports the idea that the TCE mediates translational repression. As shown in Figure $4 \mathrm{~A}$, the level of mRNA encoded by two representative tor(TCE) transgenes is similar to the level encoded by a $\mathrm{TOr}^{+}$transgene; however, the level of protein in the tor(TCE) embryos is substantially lower. Serial dilution 
Figure 4. A torso(TCE) chimeric mRNA is regulated in a manner similar to nos mRNA. (A) TCE-mediated translational repression. Shown are the results of a Northern blot (above) and a Western blot (below) of embryonic samples derived from tor $^{\mathrm{XR1}}$ females (lane 1), or samples derived from tor $^{\mathrm{XR} 1}$ females that also bear a tor $^{+}$ transgene (lane 2) or one of two representative tor(TCE) transgenes (lanes 3,4). $t^{\mathrm{XR1}}{ }^{\mathrm{I}}$ is a deletion of the entire RNA-coding region of tor. The Northern blot was hybridized with sequences homologous to tor (arrow) and, as a control, nos (arrowhead). Note that the tor(TCE) mRNA, which bears 184 additional nucleotides, migrates more slowly than the tor ${ }^{+}$ mRNA. The arrow at the bottom indicates the position of mature Tor protein; the protein detected by the antiserum that comigrates with the $120-\mathrm{kD}$ marker (indicated at the right) is present in the tor ${ }^{\mathrm{XR} 1}$ extract, and hence is not derived from tor. (B) Differential rescue of the terminalia by tor(TCE). Shown are high magnification views of the heads (left) and cuticles secreted by embryos bearing either no tor mRNA (from tor ${ }^{\mathrm{XR} 1}$ homozygous females), tor $^{+}$mRNA from one copy of a transgene (e.g., $\operatorname{tor}^{\mathrm{XR} 1} /$ tor $^{\mathrm{XR} 1}$; $\operatorname{tor}^{+}$), or tor(TCE) mRNA from one copy of a transgene. In the phase contrast photographs on the left, note that only the tor $^{+}$embryo contains structures of the anterior terminalia: labral tooth (arrow), dorsal bridge (upper arrowhead), ventral process (lower arrowhead). In the dark field photographs on the right, note that both the tor $^{+}$and tor(TCE) embryos have normal posterior terminalia including a normal eighth abdominal segment, anal pads, and filzkörper (arrow). We also constructed a tor(TCE-A) transgene (see Fig. 5) that encodes a mRNA bearing the proximal-half of the TCE. The phenotype of tor(TCE-A) embryos is similar to that shown for tor(TCE) embryos, except that some exhibit parital rescue of the anterior terminalia and some have deletions of thoracic and abdominal segments. Thus, a single copy of the CUGGC pentamer apparently is sufficient to confer differential rescuing activity on tor mRNA. Although we have not investigated the tor(TCE-A) phenotype further, our results are consistent with the idea that a single copy of the CUGGC element confers less repression than do two copies. We imagine that tor protein accumulates to intermediate levels throughout such embryos, sufficient to partially rescue anterior development but insufficient to completely trap the extracellular ligand. As a result, some of the ligand presumably diffuses toward the middle of the embryo where it ectopically activates Tor, leading to the dominant tor (or splice) phenotype. A similar phenotype arises in tor(NRE) embryos in which translation of the mRNA is inhibited in the posterior (Casanova and Struhl 1993). (C) Tor-dependent transcription of tailless (tll) is blocked at the anterior, but not at the posterior, of tor(TCE) embryos. Shown are the distributions of $t 11 \mathrm{mRNA}$ in various embryos as revealed by in situ hybridization. Embryos on the left bear wild-type mRNA from both endogenous tor $^{+}$genes, whereas embryos on the right bear tor(TCE) mRNA from a single copy of a representative transgene. The embryos in the top row are otherwise wild-type, whereas those in the middle and bottom rows lack $b c d$ and osk function, respectively. Note that the distribution of anterior $t 11$ in $(d)$ is not identical to that observed in the complete absence of Tor function (Pignoni et al. 1992), suggesting that translation of tor(TCE) mRNA is not completely inhibited. In support of this interpretation, a trace of Tor-dependent $t l l$ transcription is evident in the absence of $b c d$ function at the anterior of the embryo in $(e)$. Note also that the level of posterior $t l l$ mRNA in $(d, e)$ is somewhat less than that in $(a, b)$. This difference is attributable (at least in part) to a lower level of tor(TCE) mRNA in the embryo as compared with wild-type (see 3D). $(D)$ Localization of tor(TCE) mRNA to the pole plasm. Shown are the results of in situ hybridization with a probe homologous to tor. The wild-type embryo in $(a)$ contains tor $^{+}$mRNA from both endogenous tor ${ }^{+}$genes, and the embryo in $(b)$ contains tor(TCE) mRNA from a single copy of a representative transgene. Both are early cleavage stage embryos. No signal is detectable in control tor ${ }^{\mathrm{XR} I}$ embryos. The higher magnification views at the right are of the posterior termini of later cleavage stage embryos. Note the preferential stabilization of tor(TCE) mRNA in the pole cells.

of the protein and RNA samples suggests that translational repression reduces the level of tor protein 10- to 15 -fold. Thus, the TCE is sufficient to mediate translational repression in a mRNA bearing no other nos-derived sequences.

Examination of the cuticle secreted by tor(TCE) embryos suggested that the chimeric mRNA is translation- ally repressed at the anterior, but not at the posterior. As shown in Figure 3B, tor(TCE) embryos exhibit developmental defects of the anterior terminalia that are indistinguishable from those in $t^{-}{ }^{-}$embryos. In contrast, the posterior terminalia of tor(TCE) embryos are normal. We tested the ability of several tor(TCE) and $t r^{+}$transgenes to rescue the tor ${ }^{-}$phenotype; all of the former differen- 
tially rescue the mutant phenotype whereas all of the latter rescue both anterior and posterior defects to the same extent.

To determine whether the differential rescue of tor(TCE) embryos is the result of differential translation of the chimeric mRNA at the opposing poles of the embryo, the following experiments were performed. We were unable to visualize the distribution of tor protein directly in fixed embryos using the antibody described in Figure 3A. Therefore, expression of the zygotic gap gene tailless (tll) which is regulated by Tor activity was examined. At the posterior, transcription of tll is absolutely dependent on Tor activity, whereas at the anterior, both Bcdand Tor regulate tll transcription (Pignoni et al. 1992).

As shown in Figure 4C, insertion of the TCE into tor mRNA differentially affects $t l l$ transcription at the opposing poles of the embryo. At the anterior of tor(TCE) embryos, the level of tll mRNA is diminished and it is distributed in a broader zone, as compared with the pattern in wild-type embryos. In contrast, $t 11$ transcription at the posterior is similar in tor(TCE) and tor ${ }^{+}$embryos. These results are consistent with the idea that translation of tor(TCE) mRNA is repressed at the anterior but not the posterior of the embryo; but because of the confounding contribution of Bcd, the magnitude of this repression is difficult to assess. Accordingly, we examined the transcription of $t l l$ in tor(TCE) embryos bearing no functional Bcd. Whereas $t l l$ is transcribed in similar domains and to similar levels at both poles in $\operatorname{tor}^{+} ; b^{-} d^{-}$ embryos, we observe a strong reduction of anterior, but not posterior, tll transcription in tor(TCE); $b c d^{-}$embryos (Fig. 3C). Thus, the translational regulation of the tor(TCE) and nos mRNAs apparently is similar; both are repressed in the bulk cytoplasm and activated at the posterior pole.

We next determined whether the preferential activation of tor(TCE) mRNA at the posterior pole is dependent on Osk activity, as is the case for nos mRNA (Ephrussi and Lehmann 1992, Smith et al. 1992). In the absence of Osk function, no posterior tll transcription is observed in tor(TCE) embryos, whereas posterior $t l l$ transcription under the control of tor $^{+}$is independent of Osk function (Fig. 4C). Thus, translation of tor(TCE) mRNA is repressed in the bulk cytoplasm at the anterior and is activated in the pole plasm at the posterior; moreover, the posterior activation is Osk-dependent.

Consistent with the Osk-dependence of Tor activity in tor(TCE) embryos, tor(TCE) mRNA is concentrated in the pole plasm, much as is nos ${ }^{+}$mRNA (Fig. 3D). In contrast, tor $^{+}$mRNA is distributed uniformly throughout the bulk cytoplasm (Sprenger et al. 1989). The tor(TCE) mRNA at the posterior pole appears to be specifically associated with the pole plasm, as it is preferentially stabilized and incorporated into the pole cells; no such preferential accumulation of tor $^{+}$mRNA into the pole cells is observed (Fig. 3D).

In summary, inclusion of the TCE in tor mRNA subjects it to all three major aspects of nos mRNA regulation: translational repression in the bulk cytoplasm,
Osk-dependent relief of repression (or activation) at the posterior, and localization to the pole plasm.

\section{Tandem, redundant CUGGC pentamers mediate all three activities of the TCE}

The evidence described above demonstrates that the 184-nucleotide TCE governs translational repression, translational activation, and localization. To understand the mechanisms underlying these events, we chose to define more precisely the essential sites within the TCE. Two derivatives, nos $(\Delta \mathrm{TCE}-\mathrm{A})$ and $\operatorname{nos}(\Delta \mathrm{TCE}-\mathrm{B})$, were first constructed; these encode otherwise wild-type $\mathrm{mR}$ NAs lacking the proximal and distal half, respectively, of the TCE. Several lines of evidence support the idea that either half of the TCE is sufficient to repress the translation of unlocalized nos mRNA. First, embryos bearing either nos( $\triangle \mathrm{TCE}-\mathrm{A})$ or nos( $\Delta \mathrm{TCE}-\mathrm{B})$ mRNA are viable and exhibit none of the head defects associated with nos $(\triangle T C E)$. Second, no ectopic Nos is detectable in such embryos, and the distribution of $\mathrm{Hb}$ is essentially indistinguishable from that in wild-type embryos (data not shown). Third, biologically significant levels of ectopic Nos activity do not accumulate in osk ${ }^{-}$embryos bearing these derivatives, by the criterion that no abdominal segments develop (Fig. 5A). In contrast, the ectopic Nos activity arising from translation of unlocalized nos $(\triangle T C E)$ mRNA is sufficient to support development of a normal complement of 8 abdominal segments (Fig. 5A). Thus, the TCE apparently contains at least two redundant signals that regulate translational repression.

To further define signals within each half-TCE, we constructed five linker-scanning mutations in the region encoding TCE-A and four such mutations in the region encoding TCE-B (Fig. 5B). Sequences encoding each mutant half-TCE were substituted for those encoding the wild-type TCE, and transgenic flies were prepared. As deletion of the entire TCE causes abnormal head development and embryonic lethality (Fig. 2), the activity of each TCE mutant was assayed simply by scoring these phenotypes in embryos from the appropriate transgenic females. As summarized in Figure 5B, of the nine mutations tested, only one in TCE-A (TCE2) and two in TCE-B (TCE7 and 8) disrupt repression activity. Thus, discrete sites, one in the center of each half-TCE, appear to mediate translational repression.

Examination of the sequence in the centers of TCE-A and TCE-B reveals that each can fold into a hairpin bearing CUGGC in the loop (Fig. 5C). To test whether this pentamer is a key element in the repression signal, we constructed otherwise wild-type nos derivatives in which the sequences encoding the CUGGC pentamer in either half-TCE are substituted with sequences encoding UGCG; this sequence was chosen because it folds into a stable tetraloop in the hairpins of other RNA molecules (Woese et al. 1990), and therefore might be expected to minimally disrupt the structure of each TCE hairpin. When assayed in vivo as described above, the tetraloop mutations abolish the activity of TCE-A and TCE-B, respectively; moreover, the developmental defects in em- 
Figure 5. Tandem, redundant signals in the TCE mediate its repression activity. $(A)$ Each half of the TCE is sufficient to repress the translation of unlocalized nos mRNA. Shown are dark field micrographs of the cuticles secreted by osk ${ }^{-}$embryos that also bear the nos mRNA indicated in each panel. osk-independent rescue of abdominal segmentation constitutes a sensitive bioassay for derepression of the unlocalized nos mRNA. Note that the nos $(\triangle \mathrm{TCE}$ ) embryos (and none of the others) also have head defects not visible in the photograph. Abdominal segmentation in nos( $\triangle T C E$ ) embryos presumably is governed by a $b c d$-generated $\mathrm{Hb}$ gradient, with the ubiquitous Nos serving to block the accumulation of excess $\mathrm{Hb}$ in the posterior. The mechanisms underlying such redundant specification of abdominal segmentation are discussed elsewhere (Hülskamp et al. 1990; Struhl et al. 1992; Wharton and Struhl 1991). (B) Sequences of TCE-A and TCE-B as well as the structures of the linker-scanning mutations referred to in the text. In each case, the sequence within the bracket is substituted by the eight bases encoded by an EcoRI linker. The mutations indicated above each line abolish repression activity of the relevant half-TCE by the criterion that at least three independently derived transgenes cause dominant female sterility and head defects similar to those shown in Fig. 2 for nos( $\triangle \mathrm{TCE})$. In contrast, mutations below each line have no effect on TCE function, by the same criterion. The conserved CUGGC pentamer is highlighted. $(C)$ Critical portion of each half-TCE that can fold into a hairpin with the CUGGC pentamer comprising the loop. These hypothetical structures were identified by inspection, with guidance from the foldrna program of the GCG sequence analysis package (University of Wisconsin). Intriguingly, two copies of the CUGGC pentamer are found in the 3' UTR of the maternal CyclinB mRNA that, like nos mRNA, is localized to the pole plasm and subject to translational regulation (Dalby and Glover 1993). Perhaps the germ-line cells use a common pathway to regulate translation of the nos and CycB mRNAs.

bryos bearing a nos mRNA derivative containing both tetraloop mutations, nos $\left(\right.$ tetra $\left.^{2}\right)$, are essentially indistinguishable from those in embryos bearing nos $(\Delta \mathrm{TCE})$ mRNA (data not shown). Thus, although we have no direct evidence for the existence of these hairpins, the CUGGC pentamers appear to be essential components of the redundant signals that mediate translational repression in nos mRNA.

We next wished to determine whether the other activities mediated by the TCE- translational activation and localization- are affected by the tetraloop mutations. To this end, we constructed a derivative of $\operatorname{nos}(\Delta \mathrm{BX})$, which encodes a minimal mRNA lacking the nonessential portion of the 3' UTR (Fig. 1). (As described above, the dispensable portion of the $3^{\prime}$ UTR contains redundant signals for activation and localization which would otherwise interfere with the experiment.) This derivative, named nos $\left(\Delta \mathrm{BX}\right.$, tetra $\left.^{2}\right)$, contains mutations in the se- quences encoding both of the conserved CUGGC pentamers. The properties of these mRNAs were then examined following the preparation of transgenic flies, as described above.

Surprisingly, the tetraloop mutations also eliminate TCE-mediated translational activation and localization. As shown in Figure $6 \mathrm{a}, \operatorname{nos}(\Delta \mathrm{BX}) \mathrm{mRNA}$ is localized in a manner essentially indistinguishable from wild-type nos mRNA and is weakly translated at the posterior pole, giving rise to a very low level of protein which is barely detectable in the immediate vicinity of the posterior pole (Fig. 6b,c). In contrast, the $\operatorname{nos}\left(\Delta \mathrm{BX}\right.$, tetra $\left.{ }^{2}\right)$ mRNA is uniformly distributed throughout the cytoplasm from the beginning of embryogenesis (Fig. 6e). Moreover, we never detect an asymmetric distribution of Nos protein arising from translation of this mRNA; instead, Nos accumulates uniformly throughout the embryo (Fig. 6f,g). Apparently either the graded Nos in $\operatorname{nos}(\Delta \mathrm{BX})$ embryos 


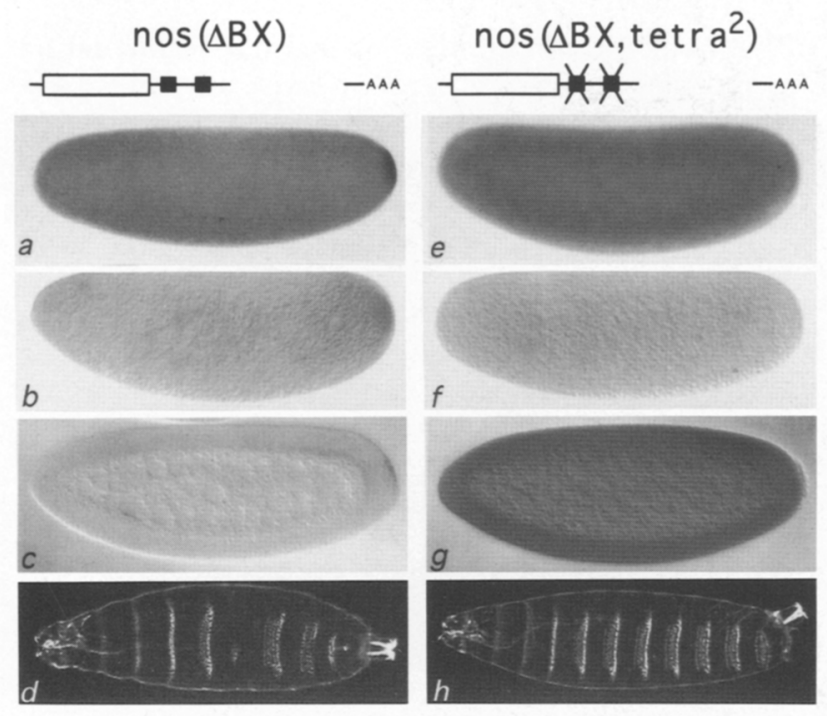

Figure 6. The CUGGC-containing signals of the TCE mediate all three of its activites. As indicated at the top, embryos on the left are derived from $\operatorname{nos}^{\mathrm{BN}}$ mutant females bearing a nos $(\Delta \mathrm{BX})$ transgene and embryos on the right are derived from nos ${ }^{\mathrm{BN}}$ females bearing a nos $\left(\Delta \mathrm{BX}\right.$, tetra $\left.{ }^{2}\right)$ transgene. Thus, all of the nos mRNA and protein in these embryos is derived from the transgene. The first row shows the distribution of nos mRNA in early cleavage stage embryos. The second and third rows show the distribution of Nos protein in early and late cleavage stages, respectively. The fourth row shows representative cuticles secreted by these embryos. Interestingly, the nos $(\Delta \mathrm{BX}$, tetra $\left.^{2}\right)_{;} n \operatorname{sos}^{\mathrm{BN}} / \operatorname{nos}^{\mathrm{BN}}$ embryos do not have head defects and develop into fertile adults. Apparently, the level of ectopic Nos in $\operatorname{nos}\left(\Delta \mathrm{BX}\right.$, tetra $\left.{ }^{2}\right)$ embryos is lower than that in nos $(\Delta \mathrm{TCE})$ embryos (Fig. 2); this lower level evidently is sufficient to allow abdominal segmentation but insufficient to interfere with anterior development. Note that the extremely low level of protein in $(b)$ is difficult to visualize, and that high concentrations of antibody and long reaction times were used in these experiments. As a result, the level of protein cannot be compared to that in embryos of Figures 2 or 3.

or the ubiquitous Nos in $\operatorname{nos}\left(\Delta \mathrm{BX}\right.$, tetra $\left.{ }^{2}\right)$ embryos is sufficient to support abdominal segmentation (Fig. 6d,h). In summary, we conclude that all three regulatory events governed by the TCE-translational repression, activation, and localization-are mediated by the same CUGGC-containing elements.

\section{Discussion}

The tethering of nos mRNA to some component of the pole plasm generally has been considered to play a preeminent role in restricting Nos activity to the posterior of the embryo. In this report, we show that three apparently distinct regulatory events-repression, activation, and localization-are intimately coupled. Within the essential portion of the nos 3' UTR, each is mediated by a pair of small, redundant signals. These observations support a model in which the Nos gradient is generated primarily by translational control and in which localization of nos mRNA is a consequence of this translational regulation rather than the converse.

A model for regulatory events at the nos TCE is presented in Figure 7. In the bulk cytoplasm, we suggest that a repressor binds to the tandem CUGGC pentamers of the TCE and thereby turns off translation of the mRNA. As development proceeds, this translationally inert mRNA is degraded. In the pole plasm, the repressor is inactivated by a localized antagonist. This antagonist may act directly by binding to the repressor (either on or off the RNA); alternatively, it may bind directly to the same pentamer-containing signals and displace the repressor. In either case, Nos protein accumulates in the posterior and the translationally active mRNA is protected from degradation. In this model, the mRNA is localized not because it is tethered to an anchor in the pole plasm, but because it is translationally activated and thereby spared from degradation. Maternal Hsp83 mRNA is thought to be localized to the posterior of the embryo by a similar mechanism: preferential degradation of mRNA in the bulk cytoplasm (Ding et al. 1993).

Critical aspects of the model described above are unt-

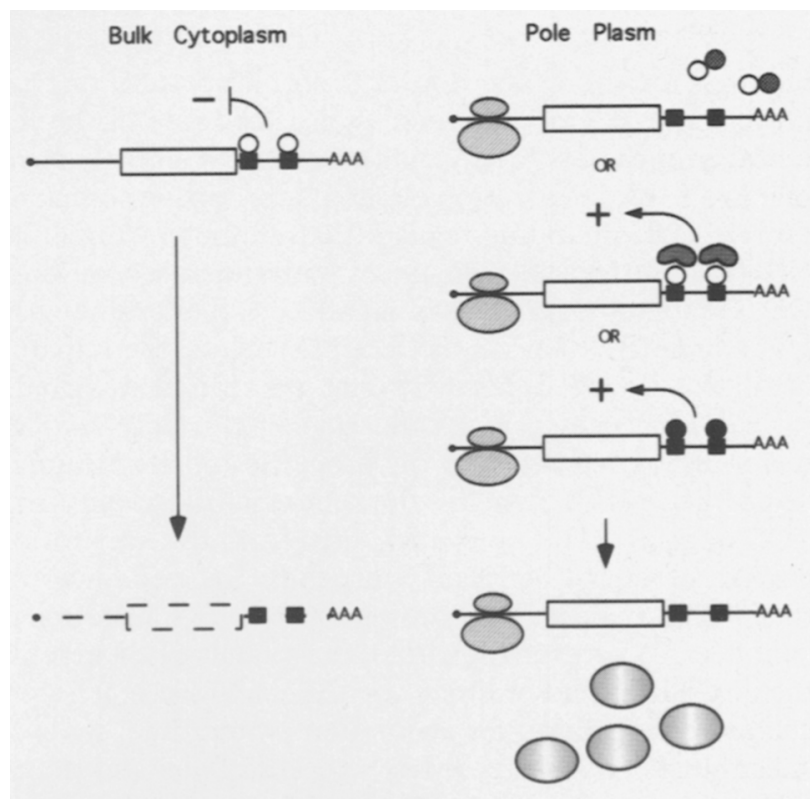

Figure 7. A model for TCE-mediated regulation of nos mRNA. The main features of the model are discussed in the text. In the bulk cytoplasm at the left, an unknown repressor $(O)$ binds to the CUGGC-containing signals in the TCE ( $\square$ ) and shuts off translation. The dashed lines indicate degradation of the RNA. In the pole plasm, an unknown activator antagonizes the activity of the repressor, and nos mRNA is translated by ribosomes (diagonal hatching) as a result. This leads to the stabilization of the mRNA and the accumulation of nos protein (shaded ellipsoids). The mechanism of activator action is unclear, and three possibilities are indicated. At the top, activation is simply relief of repression, with the liberated mRNA translated by default. In the next two scenarios, the activator simultaneously antagonizes repressor function and stimulates translation above a basal level. These latter scenarios are weakly supported by the evidence in Fig. 2. 
ested. In particular, the model assumes that translational repression is coupled to degradation of the mRNA. Consistent with this idea, we find that nos $(\triangle \mathrm{BX})$ mRNA bearing an early amber codon is undetectable, presumably because the untranslated mRNA is unstable and rapidly degraded; in contrast, nos $(\Delta \mathrm{BX})$ mRNA bearing an amber codon in the middle of the protein coding region is stable and localized normally (data not shown). Thus, premature translation termination apparently destabilizes nos $(\Delta \mathrm{BX})$ mRNA, much as has been observed in other cases (for review, see Theodorakis and Cleveland 1996). Perhaps TCE-mediated repression in the bulk cytoplasm activates the same degradation pathway, thereby contributing to the localization of the mRNA in the pole plasm.

Further tests of the regulatory model described above await the identification of the repressor and its putative antagonist, which at present are unknown. One candidate factor has been identified by Smibert and colleagues (pers. comm.), who detected in embryonic extracts a 135$\mathrm{kD}$ protein that binds specifically to the TCE. Their results, together with those presented here, are consistent with the model presented in Figure 7. However, alternative models cannot be ruled out until the $135-\mathrm{kD}$ protein, or other trans-acting factors, have been identified.

Given that the TCE appears to contain all the signals required for the generation of a normal pattern of Nos protein in the embryo, what then is the role of the remainder of the 3' UTR? Gavis et al. (1996) recently have mapped RNA localization elements throughout much of the $3^{\prime}$ UTR, including regions that are not required for Nos function (Fig. 1). Consistent with their observations, we find that nos( $\triangle \mathrm{TCE})$ is localized (albeit somewhat less efficiently than is nos ${ }^{+}$mRNA). Thus, the remainder of the 3' UTR apparently contains redundant signals for the activation and localization of the mRNA. The major difference between the properties of the minimal $\operatorname{nos}(\triangle \mathrm{BX})$ mRNA, which contains essentially only the TCE in its $3^{\prime}$ UTR, and nos ${ }^{+}$mRNA is the amount of protein produced per transcript; thus, the remainder of the 3' UTR may act simply as a nonspecific translational enhancer. Whatever their role, the signals elsewhere in the 3' UTR are subordinate to TCE-mediated repression and are not required for abdominal patterning.

For both nos and osk mRNAs, localization and translational regulation are coupled. In this report, we show that the signals that mediate repression of unlocalized nos mRNA are inseparable from signals that mediate its localization and relief of repression at the posterior pole. In contrast, the translational control and localization signals in osk mRNA are separable (Kim Ha et al. 1995). nos mRNA is localized later in oogenesis than is osk mRNA, after the antero-posterior polarity of the cytoskeleton within the egg chamber is thought to have broken down (Theurkauf 1994). Thus, it is perhaps not surprising that the mechanisms underlying regulation of these two mRNAs are somewhat different.

The importance of TCE-mediated repression is underscored by the observation that the attenuated Nos gradient generated from translation of the minimal nos $(\Delta \mathrm{BX})$
mRNA is sufficient to allow abdominal segmentation (Fig. 6). Apparently, wild-type embryos contain a large excess of Nos beyond the amount necessary to repress translation of maternal $h b$ mRNA. Taken together, these results suggest that Nos is a potent translational inhibitor. Further, they suggest that TCE-mediated repression (and the consequent degradation of nos mRNA in the bulk cytoplasm) is extremely efficient at preventing the accumulation of ectopic Nos.

A large body of evidence supports the idea that $b c d$ mRNA is the critical localized determinant for anterior embryonic patterning. By analogy, others have argued that nos mRNA is the corresponding localized determinant for posterior patterning (Gavis and Lehmann 1992; Wang et al. 1994) and that RNA localization is the primary event in establishing asymmetry (Gavis et al. 1996). The results described in this report suggest that translational regulation may play the major role in generating a restricted distribution of Nos. According to this idea, the activator of nos mRNA translation that is sequestered in the pole plasm, and not nos mRNA itself, is the key localized determinant.

\section{Materials and methods}

Strains, reagents, and techniques

The following mutant alleles were used: $\operatorname{nos}^{\mathrm{BN}}, \operatorname{tor}^{\mathrm{XR} 1}, b c d^{\mathrm{E} 1}$, $v_{a s}{ }^{\mathrm{PD}}$, and $o s k^{166}$. Wild-type flies were either Oregon $\mathrm{R}$ or $w^{118}$. Rat anti-Nos sera were described by Murata and Wharton (1995). The rabbit anti-Tor serum was a gift from F. Sprenger (University of California at San Francisco). Whole mount in situ hybridization and immunohistochemical detection of proteins were performed by standard methods. Northern blot analysis was performed using samples of total embryonic RNA; quantification was carried out using a PhosphoImager and Imagequant software (Molecular Dynamics). Total protein extracts (Sprenger and Nüsslein-Volhard 1992) were subjected to Western analysis using the ECL Detection system (Amersham).

\section{Construction of nos and tor derivatives}

The nos $^{+}$DNA used was a 5.7-kb EcoRI-DraIII genomic fragment that rescues the maternal nos mutant phenotype. A unique BamHI site was engineered using PCR immediately dowstream of the stop codon. The TCE is encoded by a 184nucleotide BamHI-BglII fragment derived from this gene. TCE-A and TCE-B are the proximal and distal halves, respectively, obtained on cleavage of the TCE coding sequences with $H p a I$. The EcoRI-BamHI fragment from the nos gene described above was fused to the MluI-XbaI fragment from the bcd 3' UTR that contains the RNA localization signals (Macdonald and Struhl 1988). nos-TCE-bcd was created by the insertion of TCE coding sequences in the BamHI site present at the junction in the nos-bcd gene. The nos $(\Delta \mathrm{TCE})$ was generated by deleting the 184-nucleotide BamHI-BglII fragment from the nos ${ }^{+}$gene described above. The tor $^{+}$DNA was an 11.5-kb EcoRI fragment that complements tor $^{-}$maternal effect lethality. Two versions of $\operatorname{tor}(\mathrm{TCE})$ that exhibited similar properties were created. In one case, TCE coding sequences were inserted into the unique SpeI site of p2599 (Wharton and Struhl 1991). In the other, TCE coding sequences were inserted at an $A s p 718$ site that was first generated using PCR immediately downstream of the Tor stop codon. For the generation of TCE $1-9$, linker scanning muta- 
tions were generated using ExoIII and Mung Bean Nuclease essentially as described by (Henikoff 1987), with deleted sequences substituted by an 8-nucleotide EcoRI linker (GGAATTCC). All of these genes were cloned into derivatives of pCaSpeR and transgenic flies were generated by P element-mediated transformation by standard methods. With a few exceptions, the phenotypes associated with at least three independent lines were examined.

\section{Acknowledgments}

We thank T. Lee and M. Patterson for excellent technical help, S. Bowling for help preparing the manuscript, F. Sprenger and T. Schüpbach for reagents and strains, C. Smibert and P. Macdonald for discussions of their related work prior to publication, and J. Heitman, X. Hou, D. Lew, D. Morisato, J. Nevins, and M. Paidhungat for helpful discussions and comments on the manuscript. Some strains were obtained from the Bloomington Stock Center. This work is supported by a grant from the Council for Tobacco Research. R.W. is an Assistant Investigator of the Howard Hughes Medical Institute.

The publication costs of this article were defrayed in part by payment of page charges. This article must therefore be hereby marked "advertisement" in accordance with 18 USC section 1734 solely to indicate this fact.

\section{References}

Casanova, J. and G. Struhl. 1989. Localized surface activity of torso, a receptor tyrosine kinase, specifies terminal body pattern in Drosophila. Genes \& Dev. 3: 2025-2038.

- 1993. The torso receptor localizes as well as transduces the spatial signal specifying terminal body pattern in Drosophila. Nature 362: 152-155.

Dalby, B. and D.M. Glover. 1993. Discrete sequence elements control posterior pole accumulation and translational repression of maternal cyclin B RNA in Drosophila. EMBO $\mathrm{f}$. 12: 1219-1227.

Ding, D., S.M. Parkhurst, S.R. Halsell, and H.D. Lipshitz. 1993. Dynamic Hsp83 RNA localization during Drosophila oogenesis and embryogenesis. Mol. Cell. Biol. 13: 3773-3781.

Driever, W. 1993. Maternal control of anterior development in the Drosophila embryo. In The development of Drosophila melanogaster, Vol. 1 (ed. M. Bate and A.M. Arias), pp. 301324. Cold Spring Harbor Laboratory Press, Cold Spring Harbor, NY.

Driever, W. and C. Nüsslein-Volhard. 1988a. A gradient of bicoid protein in Drosophila embryos. Cell 54: 83-93.

- 1988b. The bicoid protein determines position in the Drosophila embryo in a concentration-dependent manner. Cell 54: 95-104.

Ephrussi, A. and R. Lehmann. 1992. Induction of germ cell formation by oskar. Nature 358: 387-392.

Ephrussi, A., L.K. Dickinson, and R. Lehmann. 1991. oskar organizes the germ plasm and directs localization of the posterior determinant nanos. Cell 66: 37-50.

Ferrandon, D., L. Elphick, C. Nüsslein-Volhard, and D.S. Johnston. 1994. Staufen protein associates with the 3' UTR of bicoid mRNA to form particles that move in a microtubule-dependent manner. Cell 79: 1221-1232.

Gavis, E.R. and R. Lehmann. 1992. Localization of nanos RNA controls embryonic polarity. Cell 71: 301-313.

- 1994. Translational regulation of nanos by RNA localization. Nature 369: 315-318.
Gavis, E.R., D. Curtis, and R. Lehmann. 1996. Identification of cis-acting sequences that control nanos RNA localization. Dev. Biol. 176: 36-50.

Henikoff, S. 1987. Unidirectional digestion with exonuclease III in DNA sequence analysis. Methods Enzymol. 155: 156165.

Hülskamp, M., C. Pfeifle, and D. Tautz. 1990. A morphogenetic gradient of hunchback protein organizes the expression of the gap genes Krüppel and knirps in the early Drosophila embryo. Nature 346: $577-580$.

Kim-Ha, J., J.L. Smith, and P.M. Macdonald. 1991. oskar mRNA is localized to the posterior pole of the Drosophila oocyte. Cell 66: 23-35.

Kim-Ha, J., P.J. Webster, J.L. Smith, and P.M. Macdonald. 1993. Multiple RNA regulatory elements mediate distinct steps in localization of oskar mRNA. Development 119: 169-178.

Kim-Ha, J., K. Kerr, and P.M. Macdonald. 1995. Translational regulation of oskar mRNA by Bruno, an ovarian RNA-binding protein, is essential. Cell 81: 403-412.

Lieberfarb, M.E., T. Chu, C. Wreden, W. Theurkauf, J.P. Gergen, and S. Strickland. 1996. Mutations that perturb poly(A)-dependent maternal mRNA activation block the initiation of development. Development 122: 579-588.

Macdonald, P.M. and G. Struhl. 1986. A molecular gradient in early Drosophila embryos and its role in specifying the body pattern. Nature 324: 537-545.

- 1988. Cis-acting sequences responsible for anterior localization of bicoid mRNA in Drosophila embryos. Nature 336: 595-598.

Macdonald, P.M., K. Kerr, J.L. Smith, and A. Leask. 1993. RNA regulatory element BLE1 directs the early steps of bicoid mRNA localization. Development 118: 1233-1243.

Markussen, F.-H., A.-M. Michon, W. Breitwieser, and A. Ephrussi. 1995. Translational control of oskar generates Short OSK, the isoform that induces pole plasm assembly. Development 121: 3723-3732.

Murata, Y. and R.P. Wharton. 1995. Binding of pumilio to maternal hunchback mRNA is required for posterior patterning in Drosophila embryos. Cell 80: 747-756.

Nüsslein-Volhard, C., H.G. Frohnhöfer, and R. Lehmann. 1987. Determination of anteriorposterior polarity in Drosophila. Science 238: 1675-1681.

Pignoni, F., E. Steingrimsson, and J.A. Lengyel. 1992. bicoid and the terminal system activate tailless expression in the early Drosophila embryo. Development 115: 239-251.

Rongo, C., E.R. Gavis, and R. Lehmann. 1995. Localization of oskar RNA regulates oskar translation and requires Oskar protein. Development 121: 2737-2746.

Sallés, F.J., M.E. Lieberfarb, C. Wreden, J.P. Gergen, and S. Strickland. 1994. Coordinate initiation of Drosophila development by regulated polyadenylation of maternal mRNAs. Science 266: 1996-1999.

Smith, J.L., J.E. Wilson, and P.M. Macdonald. 1992. Overexpression of oskar directs ectopic activation of nanos and presumptive pole cell formation in Drosophila embryos. Cell 70: $849-859$.

Sprenger, F. and C. Nüsslein-Volhard. 1992. Torso receptor activity is regulated by a diffusible ligand produced at the extracellular terminal regions of the Drosophila egg. Cell 71: 987-1001.

Sprenger, F., L.M. Stevens, and C. Nüsslein-Volhard. 1989. The Drosophila gene torso encodes a putative receptor tyrosine kinase. Nature 338: 478-483.

St. Johnston, D. 1993. Pole plasm and the posterior group genes. In The development of Drosophila melangaster, Vol. 1 (ed. M. Bate and A.M. Arias) pp. 325-363. Cold Spring Harbor 
Laboratory Press, Cold Spring Harbor, NY.

St. Johnston, D., W. Driever, T. Berleth, S. Richstein, and C. Nüsslein-Volhard. 1989. Multiple steps in the localization of bicoid RNA to the anterior pole of the Drosophila oocyte. Dev. Suppl. 107: 13-19.

Struhl, G., P. Johnston, and P.A. Lawrence. 1992. Control of Drosophila body pattern by the Hunchback morphogen gradient. Cell 69: 237-249.

Tautz, D. 1988. Regulation of Drosophila segmentation gene hunchback by two maternal morphogenetic centres. Nature 332: 281-284.

Theodorakis, N.G. and D.W. Cleveland. 1996. Translationally coupled degradation of mRNA in eukaryotes. In Translational control (ed. J.W.B. Hersey, M.B. Mathews, and N. Sonenberg) p. 631-652. Cold Spring Harbor Laboratory Press, Cold Spring Harbor, NY.

Theurkauf, W.E. 1994. Premature microtubule-dependent cytoplasmic streaming in cappuccino and spire mutant oocytes. Science 265: 2093-2096.

Theurkauf, W.E., H. Baum, J. Bo, and P.C. Wensink. 1986. Tissue-specific and constitutive $\alpha$-tubulin genes of Drosophila melanogaster code for structurally distinct proteins. Proc. Natl. Acad. Sci. 83: 8477-8481.

Wang, C., L.K. Dickinson, and R. Lehmann. 1994. Genetics of nanos localization in Drosophila. Dev. Dynam. 199: 103115.

Wharton, R.P. and G. Struhl. 1989. Structure of the Drosophila BicaudalD protein and its role in localizing the posterior determinant nanos. Cell 59: 881-892.

. 1991. RNA regulatory elements mediate control of Drosophila body pattern by the posterior morphogen nanos. Cell 67: 955-967.

Woese, C.R., S. Winker, and R.R. Guttell. 1990. Architecture of ribosomal RNA: Constraints on the sequence of "tetraloops." Proc. Natl. Acad. Sci. 87: 8467. 


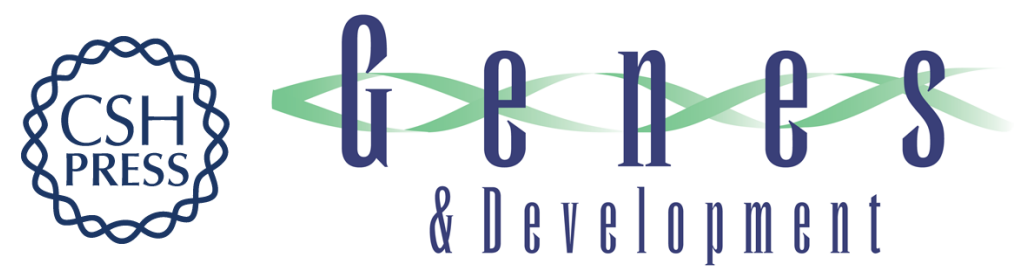

\section{The Nanos gradient in Drosophila embryos is generated by translational regulation.}

A Dahanukar and R P Wharton

Genes Dev. 1996, 10:

Access the most recent version at doi:10.1101/gad.10.20.2610

References This article cites 39 articles, 11 of which can be accessed free at: http://genesdev.cshlp.org/content/10/20/2610.full.html\#ref-list-1

License

Email Alerting Receive free email alerts when new articles cite this article - sign up in the box at the top Service right corner of the article or click here.

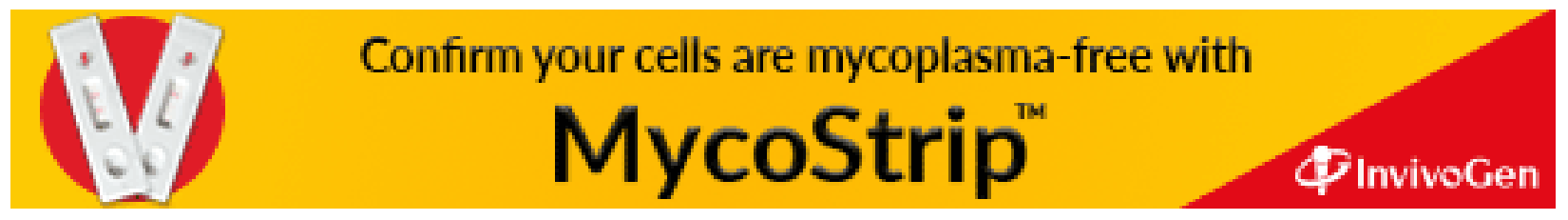

